\title{
e-Migrinter
}

$5 \mid 2010$

Migrations dans les pays nordiques

\section{« Limites et frontières des espaces israéliens et palestiniens »}

Compte-rendu du colloque international, Aix-en-Provence, les 12, 13 \& 14 novembre 2009

\section{Caroline Rozenholc}

\section{(2) OpenEdition}

\section{Journals}

Édition électronique

URL : https://journals.openedition.org/e-migrinter/1961

DOI : 10.4000/e-migrinter.1961

ISSN : 1961-9685

Éditeur

UMR 7301 - Migrinter

\section{Édition imprimée}

Date de publication : 28 avril 2010

Pagination : 70-72

ISSN : 1961-9685

\section{Référence électronique}

Caroline Rozenholc, « « Limites et frontières des espaces israéliens et palestiniens » », e-Migrinter [En ligne], 5 | 2010, mis en ligne le 20 avril 2020, consulté le 20 mai 2021. URL : http://

journals.openedition.org/e-migrinter/1961; DOI : https://doi.org/10.4000/e-migrinter.1961 


\section{« Limites et frontières des espaces israéliens et palestiniens " Colloque international, Aix-en-Provence, les 12, 13 \& 14 novembre 2009}

\section{Compte-rendu présenté par Caroline Rozenholc}

$\mathbf{E}$

n novembre dernier, et pour la deuxième année consécutive, se réunissaient en France les chercheurs et doctorants français, palestiniens, jordaniens, suisses, israéliens du projet ANR « Mobilités Frontières et Conflits dans les Espaces israélopalestiniens ». La rencontre, organisée par Stéphanie Latte Abdallah (IREMAM) et Cédric Parizot (CRFJ, IREMAM) en partenariat avec MIGRINTER, l'IREMAM, l'IDEMEC et le CRFJ, se déroulait à la Maison

Méditerranéenne des Sciences de l'Homme d'Aix en Provence. Celle-ci s'est tenue sur trois jours de colloque international intitulé : « Limites et frontières des espaces israéliens et palestiniens ", les 12 , 13 et 14 novembre 2009 .
Avec des interventions en français et en anglais, traduites simultanément, la rencontre s'est déroulée en cinq sessions, y compris l'intervention de Paolo Cuttitta de l'Université de Palerme sur une possible comparaison entre le système européen de contrôle de la migration et le "régime de frontière» entre Israël et les Territoires palestiniens (Israeli-Palestinian border regime). La journée du 12 novembre, de laquelle la conférence de P. Cuttitta participait, était inaugurée par une première intervention à deux voix. Basel Natsheh (ADTF, Ministère des Finances) et Cédric Parizot (CRFJ-IREMAM, CNRS) présentaient l'avancée de leur travail sur les trafics de marchandises entre Israël et la Cisjordanie, de 1994 à 2009. Véronique Bontemps (IDEMEC, Université Aix Marseille 1) qui intervenait sur cette même dimension économique de «la séparation» entre Israël et les Territoires palestiniens leur succédait. Véronique Bontemps présentait, elle, ses travaux sur l'expérience collective de la précarisation d'ouvriers palestiniens travaillant en Israël. Le rôle de discutante était dévolu à la sociologue Sylvie Mazella du LAMES (CNRS).

La deuxième session, intitulée «Interactions et constructions de frontières", était l'occasion de mettre en regard trois communications dont celle, pour commencer, d'Élisabeth Marteu (Paris 1 Panthéon Sorbonne). Élisabeth Marteu, spécialiste des mobilisations civiles féminines, a communiqué sur les relations 
entre Palestiniens des deux côtés de la Ligne Verte. Sylvaine Bulle de l'Université Paris Ouest Nanterre La Défense lui a succédé pour discuter des questions de mobilité dans cet espace politique fermé en abordant, en particulier, la notion de justice spatiale. Le travail qu'elle mène dans l'espace israélopalestinien se concentre en particulier sur les abords de Jérusalem. Nous lui succédions avec William Berthomière (MIGRINTER, CNRS, Poitiers) pour faire un contrepoint à la notion de frontière depuis les espaces urbains, en apportant des éléments d'analyse issus des travaux entrepris depuis 2005 sur les quartiers sud de Tel-Aviv (Neve Sheanan et Florentin). Nous développions en particulier les apports méthodologiques d'un positionnement depuis la rue - la rue comme espace d'appropriation et d'expression des identités mais aussi comme frontière - dans une réflexion sur des frontières sociales, remobilisées en Israël depuis quelques années par la présence durable de travailleurs immigrés «étrangers». Ces trois communications étaient mises en relief par Simon ImberVier du CEMAF avant d'être discutées avec les autres participants.

La deuxième journée du colloque était à son tour organisée en deux sessions. La première session sur les limites du politique nous a permis d'entendre les communications de Stéphanie Latte Abdallah (IREMAM, CNRS, Aix en Provence) sur le militantisme des prisonniers palestiniens en Israël ; de Karine Lamarche (ENS, EHESS, Paris) sur les mobilisations israéliennes contre l'occupation et le rôle que joue la frontière, et donc son passage, par les «militants infiltrés» depuis 2000 ; et, pour conclure, de Wasfi Kailani (The Royal Hashemite Court, Amman) sur les craintes qu'éveillent chez certains Musulmans les velléités de reconstruction d'un lieu de culte juif sur l'Esplanade des mosquées à Jérusalem; craintes ravivées ou entretenues par les travaux archéologiques que mènent les autorités israéliennes depuis 1967 autour du Haram al-Sharif et la médiatisation de cette question par différentes parties. Cette première session était discutée par Esmail Nashif de l'Université Ben Gurion du Negev (Beer Sheva) avant que la parole ne soit donnée à la salle.

La deuxième session «Contournements frontaliers au-delà des espaces israélo-palestiniens » était discutée par la géographe Lisa Anteby-Yemini (IDEMEC, Aix en Provence). Adoram Schneidleder (EHESS, Paris) a présenté les recherches qu'il mène sur les catégories stato-nationales en Galilée. Il a exposé le brouillage et le renforcement de ces catégories par le biais de la situation d'hommes et de femmes libanais au sud de la frontière israélo-libanaise. Ces recherches s'inscrivent d'ailleurs dans le cadre de ses travaux de doctorat sur la Galilée. Daniel Meier de l'IFPO à Beyrouth (IHEID, Genève) développait, lui, les modalités de transgression "nécessaire», de ce qu'on appelle aujourd'hui, par analogie avec la Ligne Verte entre les territoires israéliens et palestiniens, la Ligne Bleue, entre Israël et le Liban.

La journée conclusive, le samedi 14 novembre s'est organisée en deux temps, avec l'intervention de Philippe Bourmaud (Université Lyon 3, LRHRA) puis celle de Pierre Renno (ATER, Sorbonne Paris 1). La première communication exposait les effets de la désagrégation territoriale sur le système palestinien de formation aux professions de santé ; celle de Pierre Renno discutait les modalités d'application de l'ordre rationnel-légal israélien dans les territoires occupés. Sa proposition, relayée par le titre de sa communication, était d'analyser dans le détail et du point de vue des outils de la science politique, les Territoires palestiniens comme «Far-West organisationnel ». Ces deux communications ont été discutées par le sociologue Lev Grinberg de l'Université Ben Gurion du Negev, pour enchaîner avec les débats de 
clôture de cette rencontre et l'établissement du calendrier d'une prochaine rencontre. Les communications qu'ont permises ces trois journées, de même que celles présentées lors $\mathrm{du}$ premier colloque MOFIP en 2008, devraient être publiées prochainement.
Le programme du colloque et les différentes publications et événements liés au projet sont en ligne à l'adresse suivante : http://mofip.mmsh.univ-aix.fr/mofipnews/default.aspx

Caroline Rozenholc

Doctorante en géographie MIGRINTER - UMR 6588

CNRS / Université de Poitiers

ATER Université de Limoges caroline.rozenholc@etu.univ-poitiers.fr 